\title{
The Correlation between RAS and COVID-19, Short Review of the Latest Evidence
}

\author{
Antonio Vitiello, Priscilla Santilli and Francesco Ferrara* \\ Usl Umbria 1, Perugia, Italy
}

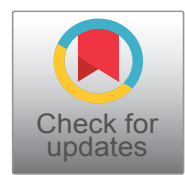

\begin{abstract}
Coronavirus SARS-CoV-2 is responsible for the Coronavirus disease (COVID-19) cause of the recent global pandemic, which is causing thousands of deaths worldwide and represents a health challenge with few precedents in human history. The angiotensin 2 conversion enzyme (ACE-2) has been identified as the receptor that facilitates access to SARS-CoV- 2 in cells; evidence shows that its concentration varies during the various stages of viral infection. Therapeutic agents modifying the renin-angiotensin system (RAS) may be able to modulate the concentration of ACE- 2 and the various components of the system. This work focuses on what has been developed to date and is known in literature. In this article we examine the latest evidence on the association between the use of RAS modifying agents and coronavirus 2019 (COVID-19) disease caused by SARS-CoV-2. Our investigation and critical literature research does not suggest discontinuation of ACEIs/ARBs treatment in clinical practice as there is a lack of robust evidence. However, we recommend further well-structured epidemiological studies investigating this sensitive issue that may provide important new suggestions for implementing guidelines.
\end{abstract}

\section{Keywords}

COVID-19, RAS, SARS-CoV-2, Pandemic

\section{Correlation SARS-CoV-2 and RAS}

The SARS-CoV-2 (COVID-19) virus is responsible for the current global pandemic, causing thousands of deaths and responsible for a health challenge with few precedents for humanity. SARS-CoV-2 is a family of RNA viruses capable of infecting humans and causing respiratory distress syndrome with severe lung injury in some fatal cases [1]. Studies have shown that SARS-Cov- 2 has about $80 \%$ of the SARS-CoV-like genome responsible for the 2003 out break [2,3]. Evidence shows that viral infection has several stages: In the first stages an asymptomatic or slightly symptomatic clinical course is described, the subsequent moderately severe stages characterised by a pulmonary inflammatory state, the last very severe stages characterised by a generalised inflammatory state affecting all tissues causing multi-organ dysfunction and in some cases death [4]. Biochemical interaction studies have shown that SARS-CoV-2 virus enters host cells mainly through the use of the spike protein $(S)[5,6]$ through the angiotensin 2 conversion enzyme receptor (ACE-2) on the cell surface [6]. ACE-2 is also a conversion enzyme that is part of the renin-angiotensin system (RAS). Is there a scientific debate going on since the beginning of the pandemic, is an increase of ACE2 responsible for a higher probability of COVID-19 infection? Lung tissues are probably an easier entry route for SARS-CoV2 because $83 \%$ of ACE-2 receptors are present in type II pneumocytes that produce surfactants that prevent to the alveoli to collapse [7-14]. The RAS modulating drugs are widely used in the treatment of cardiovascular diseases, but what is the correlation between these drugs, RAS and COVID-19? Can they play a protective role by modulating the expression of RAS components? Or, on the contrary, are these drugs considered risk factors for COVID-19?

\section{Recent Evidence on the Topic}

To date, it is strongly recommended not to interrupt the treatment with the usual therapy of RAS modulating drugs, because no clinical or scientific evidence suggests this [15]. Agents acting on RAS can be distinguished as inhibitors of the angiotens in conversion enzyme (ACEI), Angiotensin II receptor blockers (ARB) and direct renin inhibitors (DRI) [16]. These agents are currently indicated for the treatment of various cardiovascular diseases with excellent clinical efficacy. ACEIs are able to reduce blood pressure by acting with ACE inhibition which converts Ang I to Ang II. ARBs are Ang II antagonists on the type 1 receptor (AT-1r), finally, DRIs block plasma

*Corresponding author: Dr. Francesco Ferrara, UsI Umbria 1, Perugia, Italy

Accepted: October 09, 2020

Published online: October 11, 2020

Citation: Vitiello A, Santilli P, Ferrara F (2020) The Correlation between RAS and COVID-19, Short Review of the Latest Evidence. Ann Public Health Reports 4(1):73-75 
renin activity and inhibit the conversion of angiotensinogen to Ang I. The three different classes described above have different effects on the regulation and enzymatic expression of RAS [17]. A retrospective epidemiological cohort study using database data showed that the use of ACEI or ARB was not significantly associated with mortality and diagnosis of COVID-19, respectively [18]. Another study using electronic health records from the University of New York (NYU) Langone Health showed no significant correlation and association between the use of ACEI/ARB and the development of COVID-19 and COVID-19 severe, respectively [19]. In addition, another retrospective and multi-centre study conducted on a large scale in adult hypertensive patients with COVID-19 in Hubei, China 9, showed that the use of ACEI or ARB was significantly associated with a lower probability of mortality due to different causes than non-users of ACEI/ARB, probably for a greater and more effective management of underlying cardiovascular disease in the patients considered. Finally, some studies have not been considered because they show inconsistent data [20]. In addition, in several studies, investigations in patients receiving ACEi or ARB treatment did not have higher plasma concentrations and significant changes in ACE-2, in contrast to in vitro data [21]. In conclusion, based on currently available data and taking into account evidence of reduced mortality in cardiovascular disease, ACE-I and ARB therapy should be maintained or initiated in patients with cardiovascular disease according to current guidelines of the major scientific societies.

\section{Conclusion}

The Covid-19 pandemic has not yet ended, but these mechanisms described have been shown to be the key to action to defeat the current pandemic. If a vaccine is still a long way off, the possibility of developing drugs that act at the level of the RAS system can certainly be a winning breakthrough. Surely in a short time other work will clarify the feasibility of a cure that can permanently curb the virus and free the whole of humanity.

\section{Conflicts of Interest}

None of the authors have conflicts of interest to disclose.

\section{Funds}

None.

\section{Copyright}

The authors certify that the manuscript is original, never submitted to other journal for publication before. All authors contributede qually to the manuscript and had the opportunity to revise and approve the final text.

\section{Disclosure Statement}

\section{Dr. A.Vitiello has nothing to disclose.}

Dr. F. Ferrara has nothing to disclose.

\section{Acknowledgement}

The authors have nothing to say about ethical standards, ethical approval and funding. This manuscript is not a clinical trial and does not violate ethical rules. No funding has been received for its preparation.

\section{References}

1. Baig AM, Khaleeq A, Ali U, et al. (2020) Evidence of the COVID-19 virus targeting the CNS: Tissue distribution, host-virus interaction, and proposed neurotropic mechanisms. ACS Chem Neuroscienc 11: 995-998.

2. Liu Z, Xiao X, Wei X, et al. (2020) Composition and divergence of coronavirus spike proteins and host ACE- 2 receptors predict potential intermediate hosts of SARS-CoV-2. J Med Virol 92: 595601.

3. Cascella M, Rajnik M, Cuomo A, et al. (2020) Features, evaluation and treatment Coronavirus (COVID-19). StatPearls [Internet]. Treasure Island (FL): StatPearls Publishing.

4. Ashour HM, Elkhatib WF, Rahman MM, et al. (2020) Insights into the recent 2019 novel Coronavirus (SARS-CoV-2) in light of past human Coronavirus outbreaks. Pathogens 9: 186.

5. Yan R, Zhang Y, Li Y, et al. (2020) Structural basis for the recognition of the SARS-CoV-2 by full-length human ACE2. Science 367: 1444-1448.

6. Walls AC, Park YJ, Tortorici MA, et al. (2020) Structure, function, and antigenicity of the SARS-CoV-2 spike glycoprotein. Cell 181: 281-292.

7. Sharma Manik, Samriti Sharma (2020) The rising number of COVID-19 cases reflecting growing search trend and concern of people: A google trend analysis of eight major countries. J Med Syst 44: 117.

8. Sharma Samriti, Manik Sharma, Gurvinder Singh (2020) A chaotic and stressed environment for 2019-nCoV suspected, infected and otherpeople in India: Fear of mass destruction and causality. Asian J Psychiatr 51: 102049.

9. Gautam Ritu, Manik Sharma (2020) 2019-nCoV pandemic: A disruptive and stressful atmosphere for Indian academic fraternity. Brain Behav Immun 88: 948-949.

10. Bonaccorsi Giovanni, Francesco Pierri, Matteo Cinelli, et al. (2020) Economic and social consequences of human mobilityrestrictions under COVID-19. Proceedings of the National Academy of Sciences 117: 15530-15535.

11. Ludvigson Sydney C, Sai Ma, Serena Ng (2020) Covid19 and the macroeconomic effects of costly disasters. National Bureau of Economic Research Paper No. w26987.

12. Sher Leo (2020) The impact of the COVID-19 pandemic on suicide rates. QJM: An International Journal of Medicine.

13. Douglas Margaret, Srinivasa Vittal Katikireddi, Martin Taulbut, et al. (2020) Mitigating the wider health effects of covid-19 pandemic response. BMJ 369: $\mathrm{m} 1557$.

14. Monga Preeti, Manik Sharma, Sanjeev Kumar Sharma (2020) Need of surveillance system to support digital psychological intervention to COVID19 afflicted people in India. International Journal of Social Psychiatry.

15. Zhang H, Penninger JM, Li Y, et al. (2020) Angiotensin-converting enzyme 2 (ACE2) as a SARS-CoV-2 receptor: Molecular mechanisms and potential therapeutic target. Intensive Care Med 46: 586-590.

16. Bavishi C, Bangalore S, Messerli FH (2016) Renin angiotensin al- 
dosterone system inhibitors in hypertension: Is there evidence for benefit independent of blood pressure reduction? Prog Cardiovasc Dis 59: 253-261.

17. Antonio Vitiello, Francesco Ferrara (2020) Correlation between renin-angiotensin system and severe acute respiratory syndrome coronavirus 2 infection: What do we know? Eur J Pharmacol 883: 173373.

18. Fosbø| EL, Butt JH, Østergaard L, et al. (2020) Association of angiotensin-converting enzyme inhibitor or angiotensin receptor blocker use with COVID-19 diagnosis and mortality. JAMA 324 168-177.
19. Reynolds HR, Adhikari S, Pulgarin C, et al. (2020) Renin-angiotensin-aldosterone system inhibitors and risk of COVID-19. N Engl J Med 382: 2441-2448.

20. Zhang P, Zhu L, Cai J, et al. (2020) Association of inpatient use of angiotensin converting enzyme inhibitors and angiotensin II receptor blockers with mortality among patients with hypertension hospitalized with COVID-19. Circ Res 126: 1671-1681.

21. Sama IE, Ravera A, Santema BT, et al. (2020) Circulating plasma concentrations of angiotensin-converting enzyme 2 in men and women with heart failure and effects of renin-angiotensin-aldosterone inhibitors. Eur Heart J 41: 1810-1817. 